Proceedings

\title{
Tuning the Crystallizability of Renewable Poly(alkylene 2,5-furan-dicarboxylate)s by In-Situ Adding Various Nanosized Nucleation Agents ${ }^{+}$
}

Panagiotis A. Klonos 1,2,*, Lazaros Papadopoulos 1, Zoi Terzopoulou 1, Apostolos Kyritsis 2, George Z. Papageorgiou ${ }^{3}$ and Dimitris N. Bikiaris ${ }^{1, *}$

1 Department of Chemistry, Laboratory of Polymer Chemistry and Technology, Aristotle University of Thessaloniki, GR-541 24, Thessaloniki, Greece; lazaros.geo.papadopoulos@gmail.com (L.P.); terzoe@gmail.com (Z.T.)

2 Department of Physics, National Technical University of Athens, Zografou Campus, 15780, Athens, Greece; akyrits@central.ntua.gr

3 Laboratory of Industrial and Food chemistry, Chemistry Department, University of Ioannina, 45110 Ioannina, Greece; gzpap@cc.uoi.gr

* Correspondence: pklonos@central.ntua.gr (P.A.K.); dbic@chem.auth.gr (D.N.B.); Tel.: +30-2310-997812

+ Presented at the First International Conference on “Green” Polymer Materials 2020, 5-25 November 2020; Available online: https://cgpm2020.sciforum.net/.

Published: 4 November 2020

\begin{abstract}
Poly(alkylene 2,5 furan-dicarboxylate)s (PAFs) belong in a relatively new class of biobased polyesters prepared from renewable resources aiming at use in food packaging applications, owing to their excellent mechanical and gas barrier performance. Both properties are severely connected to crystallinity. Thus, the enhancement of the latter is attempted here by the in situ introduction in the PAF matrix of a variety of inorganic nanofillers, graphene nanoplatelets, carbon and halloysite nanotubes, nanoclays and silica nanoparticles. The nanofillers are used in the bulk/initial state as well as upon surface modifications, moreover, in the forms of mixtures and hybrid particles. For the structural and crystallization investigation, we employ the following combination of techniques: Fourier transform infra-red spectroscopy, calorimetry, X-ray diffraction, polarized optical microscopy and broadband dielectric spectroscopy (BDS). The direct effects of the fillers on the rate as well as the amount of crystallization are correlated with the semicrystalline morphology, with molecular mobility and, interestingly, with the mechanical performance. The employed fillers facilitate crystallization, acting as additional nuclei, with the strength of this effect increasing systematically with the specific surface area and/or the aspect ratio of the fillers. Contrariwise, upon surface modification and development of strong polymer-particle interactions the nucleating action is hindered, which is demonstrated in PAFs for the first time. As expected, crystallization, semicrystalline morphology and interfacial interactions impose strong impacts on molecular dynamics (BDS). Overall the results suggest that such materials serve quite well as tailormade nanocomposites for targeted applications.
\end{abstract}

Keywords: poly(alkylene 2,5-furan-dicarboxylate); polymer crystallization; nanocomposites 


\section{Introduction}

Polymers and polymer nanocomposites (PNCs) are used in a wide range of applications in everyday life, industry and academia, mostly due to their low production costs and the good performance in various wanted properties [1]. PNCs demonstrate tremendously improved properties [1] as compared to the unfilled polymers and the macro-composites [2], owing mainly to large surface to volume ratio of the used nano-inclusions [1,3]. Recently, our world has come in front of serious environmental concerns, including the use of commodity plastics, the latter being prepared from finite resources and exhibiting non eco-friendly character, e.g., extremely slow biodegradability, hazardous recycling routes. Solution to that has been found via the development and application of biodegradable polymers during the last decade. The biobased polyesters produced from 2,5-furandicarboxylic acid (FDCA), a renewable building block derived from 5-hydroxymethylfurfural sources, known as poly(alkylene 2,5 furan-dicarboxylate)s (PAF) apart a relatively new class of materials targeted to replace their fossil-based homologues quite soon [4]. PAFs have been already considered ideal candidates for packaging applications, owing to their excellent gas barrier properties and good mechanical performance.

Herein, we demonstrate the potentials for tuning the crystallizability of three PAFs, poly(propylene-/butylene-/hexylene- funoates) (PPF/PBF/PHF), by reinforment the polymer via the in situ introduction of low amounts of various nanofillers of different geometries, aspect ratio (AR) and surface modifications. We investigate the impact of the fillers addition on the rate and amount of crystallization, the semicrystalline morphology and the molecular mobility, namely glass transition, segmental and local dynamics. To that aim, we have employed a sum of complementary structure and thermo-dynamical methods, being described in the following.

\section{Experiments}

\subsection{Materials}

PPF, PBF and PHF were synthesized by a known two-stage process, involving transterification and polycondensation [5], more specific details on the PAFs synthesis being available in previous work [6-8]. For the PNCs, the fillers were inserted in the matrix in situ simultaneously with the polymer synthesis. The chosen nanofillers for PAFs are: montomorillonite (MMT) nanoclays, natural (MMT-Na) and of two modifications (MMT-DD, MMT-DBe), halloysite nanotubes (HNT), carbon nanotubes $(\mathrm{CNT})$ and modified CNT (modCNT), graphene (Gr), graphene oxide (GO) and modified GO (modGO), silica $\left(\mathrm{SiO}_{2}\right)$, as well as mixed $\mathrm{Gr}$ and $\mathrm{SiO}_{2}$ particles (mixGrSi). The filler loading was chosen low for all cases, namely in the range $0.5-2.5 \mathrm{wt} \%$.

\subsection{Experimental Methods}

Differential scanning calorimetry (DSC), X-ray diffraction (XRD), polarized light microscopy (POM) were employed to investigate crystallization and thermal transitions, while via Fourier transform infra-red (FTIR) spectroscopy the interfacial filler-polymer interactions were assessed and evaluated. Finally, the high resolving power technique of broadband dielectric spectroscopy (BDS) [9] was employed to investigate polymer dynamics of the segmental (glass transition) as well as the local type, in a wide range of temperatures and frequencies.

\section{Results and Dicussion}

The crystallization of PAFs is in general slow as compared to other polymers and this is due to the 'heavy' furan ring at the polymer backbone [4,5]. This results initially in the hindering of both the nucleation, related also with the physical crosslinking [10] (and references therein), and, moreover, of the chain foldings, the latter being necessary for the crystalline lamellae formation (crystals growth). Therefore, for a given PAF structure, the poor nucleation can be improved by properly chosen nucleating agents, which in our case are inorganic nanofillers. 


\subsection{Crystallization}

In Figure 1 it can be directly seen that the in situ addition of initial nanofillers impose the direct effect of accelerating nucleation of PAFs. Interestingly the most strong effects of improving nucleation is recorded for CNTs, nanoclays and GO, which are actually the fillers exhibing quite large aspect ratios $[3,11]$ and specific surface areas. Despite the increase in the rate of crystallization, the fraction of crystals (CF) does not change significantly from the unfilled matrix to the PNCs. It should be noted that CF of PAFs is not tremendously high, as it varies between $\sim 12$ and $\sim 35 \mathrm{wt} \%$. Combining these results together, it can be easily concluded that the presence of the nucleating-agent fillers should impose serious alternations in the semicrystalline morphology, namely the number (increased) and size (decreased) of the formed crystals. This has been checked by POM and is shown here at the example of PBF filled with MMTs in Figure 2.

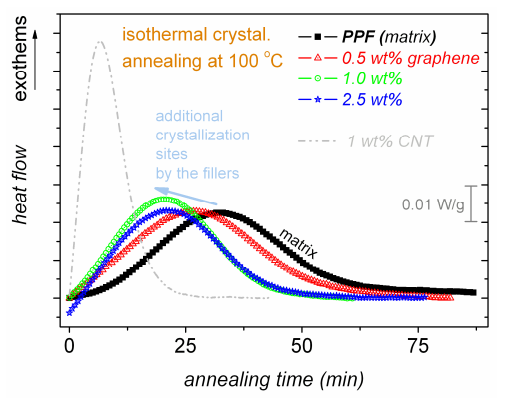

(a)

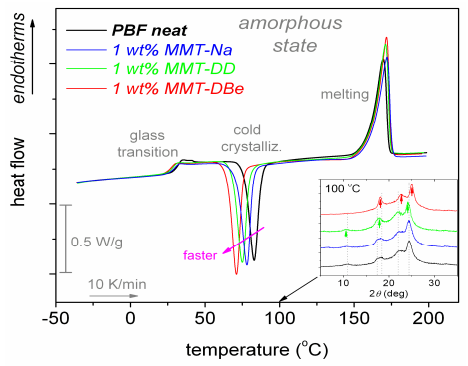

(c)

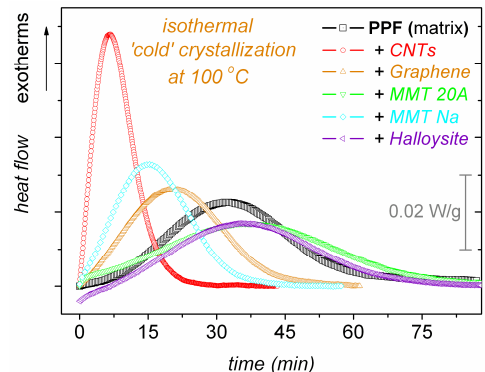

(b)

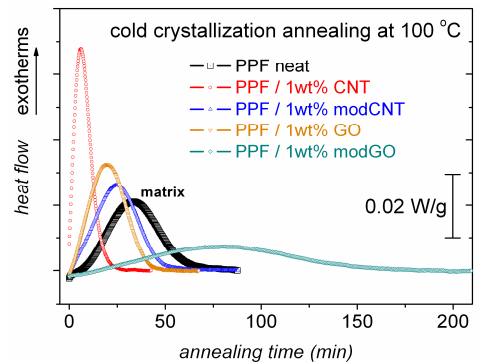

(d)

Figure 1. $(\mathbf{a}-\mathbf{c})$ Comparative DSC thermograms showing the effects of various initial nanofillers on crystallization of PAFs (isothermal crystallization or cold crystallization during heating). The inset to (c) shows the corresponding results by XRD. (d) Effects of filler surface modification (stronger interfacial interactions) on the rate of crystallization of PPF.
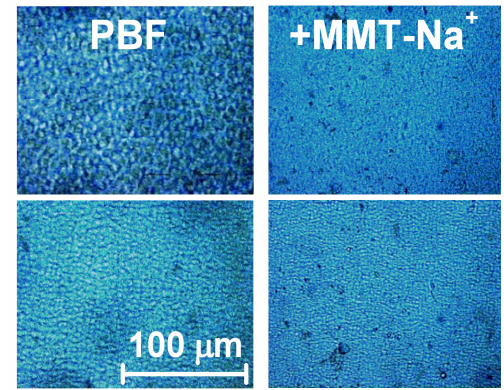
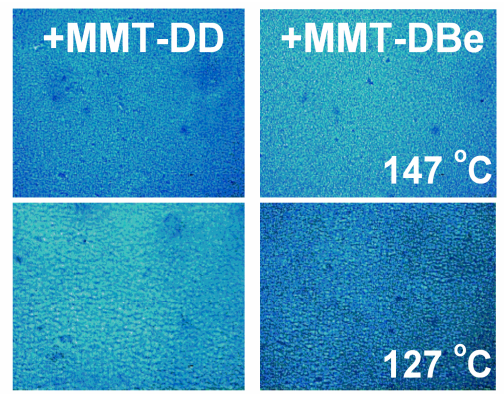

Figure 2. POM images of PBF-based systems for different crystallization conditions/temperatures.

Additional support for the expected alternations in the semicrystalline morphology is supplied by the XRD data of Figure 3, wherein, the diffraction peaks arising from the polymer spherulites are in many cases of PNCs located at different $2 \theta$ positions as compared to the corresponding neat PAF 
matrix. Also, the data by XRD have enabled the evaluation of $\mathrm{CF}$ upon proper analysis of the complex spectra. CF by XRD is in most cases in quite good agreement with CF estimated by DSC.

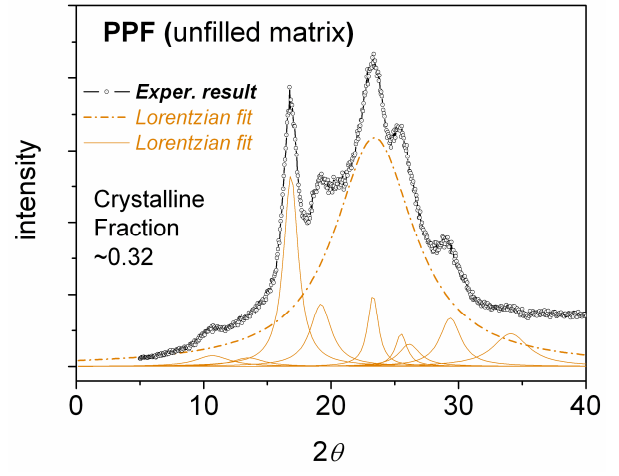

(a)

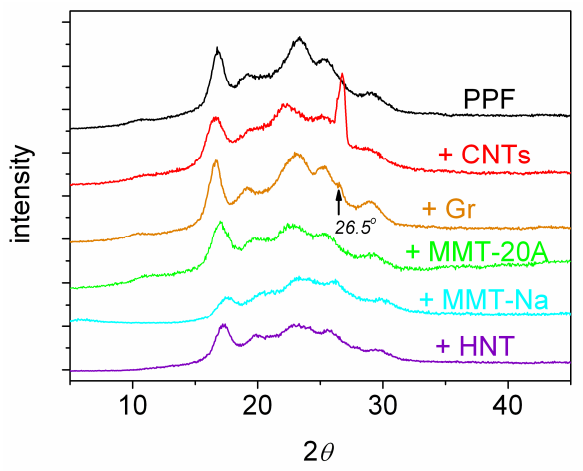

(c)

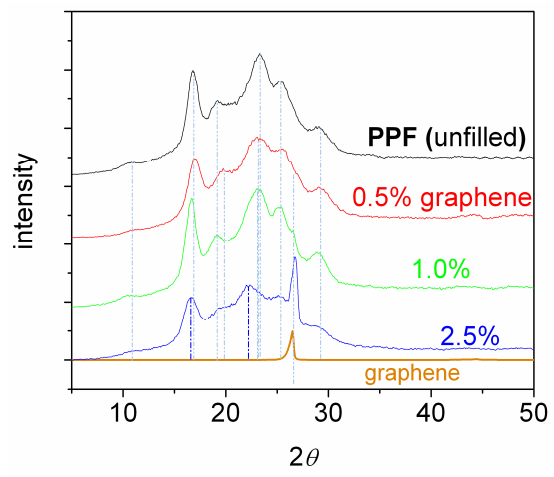

(b)

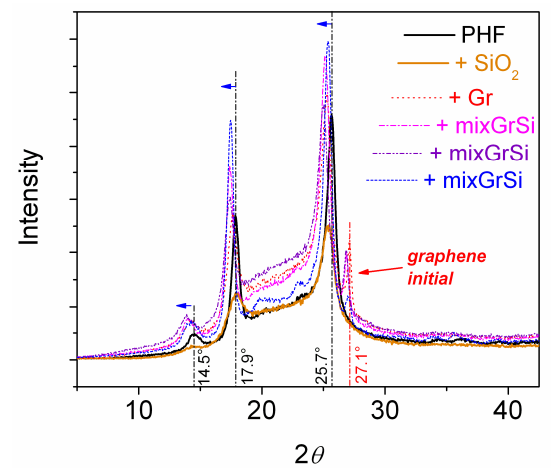

(d)

Figure 3. Comparative XRD spectra for the various samples indicated on the plots. (a) shows a result of analysis of the spectrum in terms of individual Lorentzian peaks at the example of unfilled PPF.

\subsection{Polymer-Filler Interactions}

In Figure 4 we show the comparative FTIR spectra for selected samples, in the wavenumber range around $1725 \mathrm{~cm}^{-1}$, which is related with the disturbance of the ester bonds of PAF. These bonds are expected to be more seriously involved within potential interfacial interactions [12].

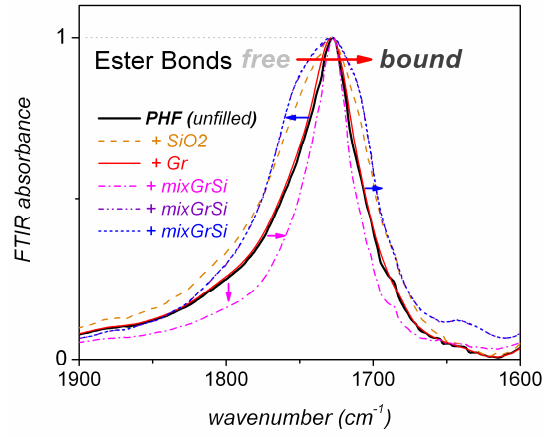

(a)

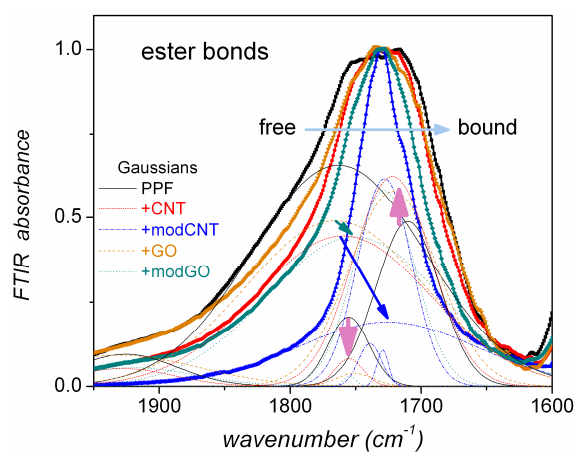

(b)

Figure 4. Comparative FTIR spectra for (a) PHF and PHF-based nanocomposites of $\mathrm{SiO}_{2}$, $\mathrm{Gr}$ and mixGrSi and (b) PPF and nanocomposites with initial and surface modified CNT and GO. The FTIR peaks of main interest are shown, namely those related to the free and disturbed (bound) ester bonds, as representatives of the interfacial polymer-filler interactions. 
In the cases of initial (unmodified) fillers (Figure 4a), the said peaks do not demostrate serious disturbances, suggesting weak interfacial interactions. On the other hand, the involvement of surface modifications of the fillers impose severe disturbances of the FTIR peak (Figure $4 b$ ), in particular, migrations of the said peaks toward lower wavenumbers in the PNCs. This is indicative of transition from 'free' to 'bound' groups due to interactions $[12,13]$. The latter case of modified CNTs and GO, has been interpreted by the formation of a 'bound' interfacial polymer layer around the fillers, the presence of which precludes or hinders the crystal nucleation around these fillers. The latter is documented by the crystallization deccelaration in Figure 1d, comparing with the PNCs containg non-modified fillers. This effect on nucleation is demonstrated here [12] for the first time, to the best of our knowledge.

\subsection{Effects on the Mechanical Performance}

Coming to the initial scope of the PNCs synthesis, i.e., the improvement of the mechanical performance to make the PAF-based materials more suitable for the wanted applications, we turn the focus on Figure 5. Therein, we show the changes in the elastic modulus upon the addition of various fillers. The direct filler effects on the mechanical performance is more obvious at the absence of crystals. However, the changes in the elastic modulus are not straightforward (Figures 5b,c), despite an on average moderate increasing trend. This can be understood via the quite low filler loadings $(0.5-2.5 \mathrm{wt} \%)$.

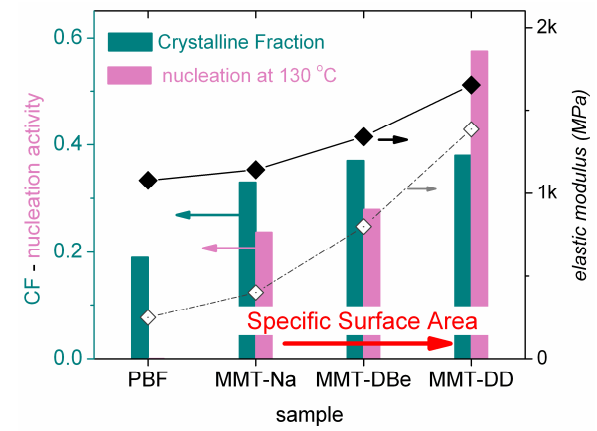

(a)

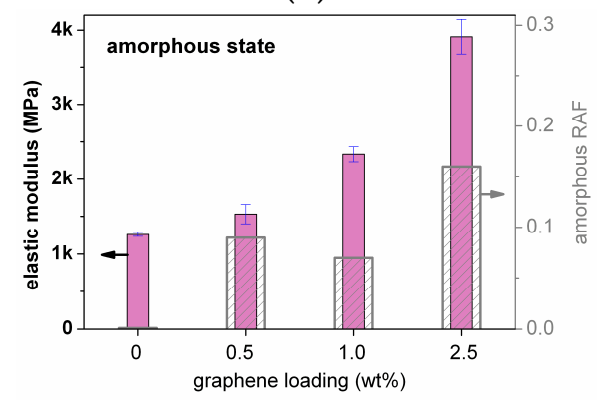

(c)

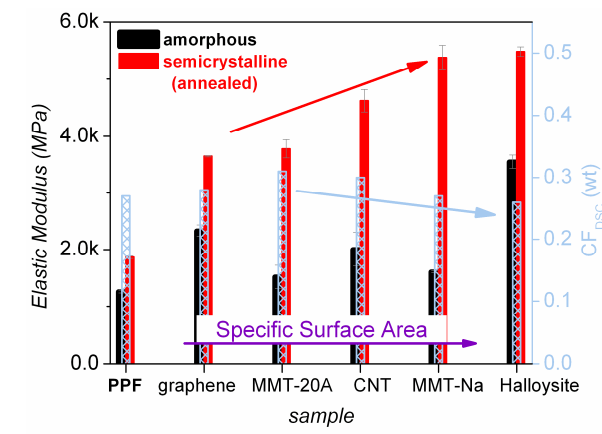

(b)

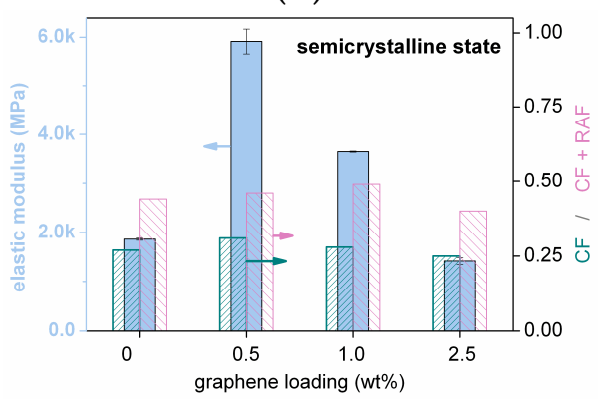

(d)

Figure 5. Correlation of the mechanical performance of the various PAF-based nanocomposites (described on the plots) with the improved CF, RAF (interfacial fraction) and the surface characteristics of the employed fillers. For the PPF/Gr systems, (c) and (d) show the changes between the amorphous and the semicrystalline state of the polymer, respectively.

As expected, the most serious effects on the elastic modulus arise from the improvements in crystallinity. Interestingly, one would expect the mechanical enhancement to coincide with the increase in $\mathrm{CF}$ and increase in ' $\mathrm{CF}+\mathrm{RAF}^{\prime}$ (interfacial rigid polymer fraction) [14]. This is not recorded here in the PAFs-based PNCs, as CF is mainly not increasing. Interestingly, there is indeed a significant mechanical improvement that arises actually from the presence of many small crystals in the PNCs as compared to the fewer but larger crystals in the unfilled PAF matrix. The latter effect 
resembles the situation between the conventional polymer macro-composites (macroscopic fillers) and polymer nano-composites (nano-sized fillers), as the surface to volume ratio in the latter is significantly larger [2]. This is a quite interesting point regarding the mechanical improvement in semicrystalline PNCs, being also useful from the point of view of PNC processing. Similar effects on however the thermal conduction improvements arising from the special semicrystalline morphology have been recently demonstrated for other PNCs based on conventional polymers $[15,16]$.

\subsection{Effects on the Molecular Dynamics}

In the last section of this article we focus on moleculer dynamics, as recorded by the more advanced technique of BDS. The BDS results have been carefully/critically analysed and are summarized in the overall dielectric maps (Arrhenius plots) [9] of Figure 6.

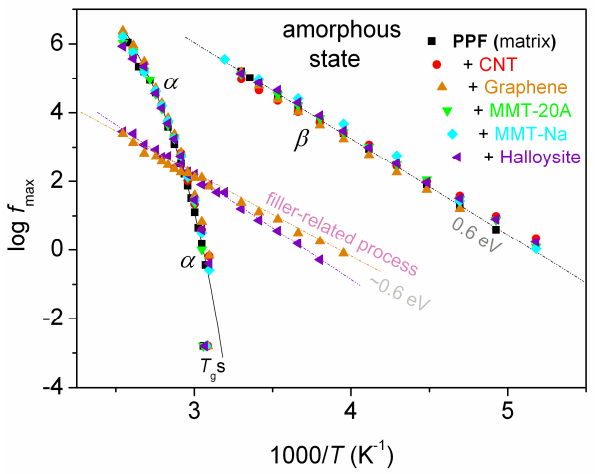

(a)

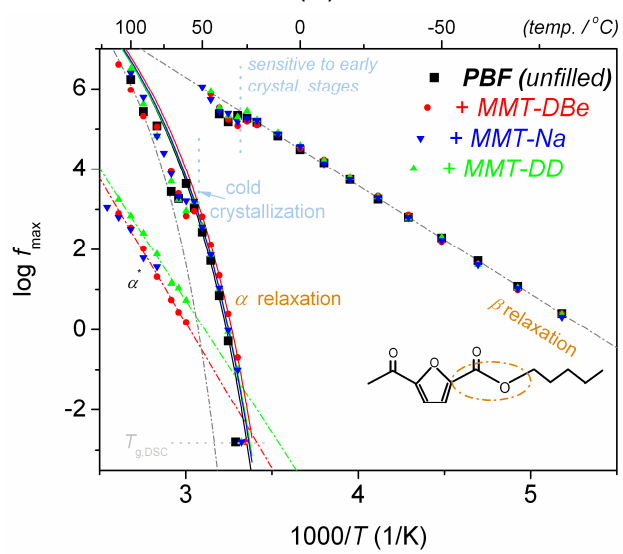

(c)

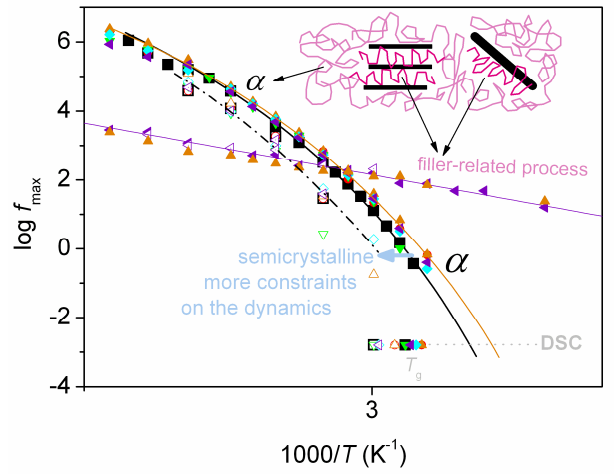

(b)

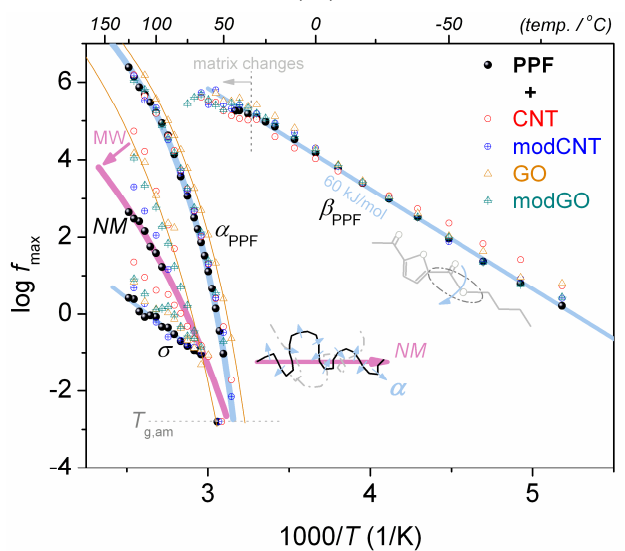

(d)

Figure 6. Molecular dynamics maps (Arrhenius plots) for the various PAF-based systems decribed therein, showing the local $(\beta)$, segmental $(\alpha)$, global chain $(\mathrm{NM})$, filler-related $\left(\alpha^{*}\right)$ processes and conductivity relaxation $(\sigma)$. For comparison with DSC, the respective calorimetric $T_{\mathrm{g}}$ points have been added at the corresponding equivalent frequency $(\log f \sim-2.8)$. The added lines connecting the experimental data are fittings of the Arrhenius (straight lines) and Vogel-Fulcher-Tammann-Hesse (curved lines) equations. The origins of the different processes are described via the inset schemes.

At the lower temperatures (Figure $6,-100$ to $+50{ }^{\circ} \mathrm{C}$ ), the local $\beta$ relaxation process is recorded for all samples. $\beta$ arises from crankshaft motions of the molecular group related to the chemical link between the aromatic ring and the ester carbon (inset scheme) [17]. As expected it does not change for the different fillers, however interestingly seem to sense large scale thermal transition of the matrix (crystallization, glass transition). At higher temperatures, namely $T>T_{\mathrm{g}}$, the segmental $\alpha$ process is recorded, which is the dielectric analogue of the glass transition. $\alpha$ is a cooperative relaxation and in the PAFs decelerates with the development of crystallization (Figures $6 b, c)$. In the amorphous state and for low molecular weights (MW $<20 \mathrm{~kg} / \mathrm{mol}$ ), $\alpha$ accelerates when the MW drops 
(Figure 6d). For the latter case of low MW the Normal Mode (NM) process is recorded. NM reflects the chain end-to-end vector movement, namely the global chain dynamics (Figure 6d). Regarding the filler effects, in some cases of fillers (Gr, HNT, MMT) additional filler related processes are recorded, both faster and slower as compared to $\alpha$ process (Figures 6a-c). These processes have been proposed to arise from the polymer chains either directly adsorbded at the outer filler surface or confined between nanoplatelets, whereas within that dynamics the role of water traces (e.g., in MMT) has been found important [7].

\section{Conclusions}

We investigated the impact of various nanofillers on the crystallization, mechanical performance, interfacial interactions and molecular dynamics of PAFs (PPF, PBF, PHF). All fillers were in situ dispersed throughout the polymer matrix. Despite the extremely low filler loadings of $0.5-2.5 \mathrm{wt} \%$, all fillers were found to accelerate crystallization, offering additional crystallization sites (DSC). This improvement was found to correlate with the fillers' specific surface area. CF of neat PAFs, on the other hand, was not increased, suggesting alternations in the same crystalline morphology, i.e., smaller but many more crystals in the PNCs (POM, XRD). Due to the latter effect, the fillers were found to impose an indirect effect on the mechanical performance increasing the eleastic modulus. The implementation of increased interfacial interfacial interaction, achieved via surface modifications of the fillers (CNT, GO) resulted in opposite effects on crystallization (slower), which is shown here for the first time. Glass transition (DSC) and segmental dynamics (BDS) were found to change indirectly with the filler presence, i.e., via changes in the crystallization and/or the drop in MW. The local $\beta$ dynamics was found to be sensitive to the early stages of crystallization, whereas in some PNCs additional dynamics processes were recorded, most probably arising from the polymer dynamics in the vicinity of the particles. Overall, these systems provide high possibilities for tailor-making systems, also envisaging their employment in specific applications.

Author Contributions: Conceptualization, D.N.B. and P.A.K., L.P.; methodology, L.P., P.A.K. and D.N.B.; validation, A.K., Z.T., G.Z.P. and D.N.B.; formal analysis, P.A.K.; investigation, P.A.K., L.P., and Z.T.; resources, A.K., and D.N.B.; writing - original draft preparation, P.A.K.; writing-review and editing, P.A.K., and D.N.B.; supervision, D.N.B. All authors have read and agreed to the published version of the manuscript.

Acknowledgments: This publication is based upon work from COST Action FUR4Sustain, CA18220, supported by COST (European Cooperation in Science and Technology).

Conflicts of Interest: The authors declare no conflict of interest.

\section{References}

1. Paul, D.R.; Robeson, L.M. Polymer nanotechnology: Nanocomposites. Polymer 2008, 49, 3187-3204, doi:10.1016/j.polymer.2008.04.017.

2. Bokobza, L.; Chauvin, J.P. Reinforcement of natural rubber: Use of in situ generated silicas and nanofibres of sepiolite. Polymer 2005, 46, 4144-4151, doi:10.1016/j.polymer.2005.02.048.

3. Raquez, J.M.; Habibi, Y.; Murariu, M.; Dubois, P. Polylactide (PLA)-Based nanocomposites. Prog. Polym. Sci. 2013, 38, 1504-1542, doi:10.1016/j.progpolymsci.2013.05.014.

4. Papageorgiou, G.Z.; Papageorgiou, D.G.; Terzopoulou, Z.; Bikiaris, D.N. Production of bio-based 2,5-furan dicarboxylate polyesters: Recent progress and critical aspects in their synthesis and thermal properties. Eur. Polym. J. 2016, 83, 202-229, doi:10.1016/j.eurpolymj.2016.08.004.

5. Papageorgiou, G.Z.; Papageorgiou, D.G.; Tsanaktsis, V.; Bikiaris, D.N. Synthesis of the bio-based polyester poly(propylene 2,5-furan dicarboxylate). Comparison of thermal behavior and solid state structure with its terephthalate and naphthalate homologues. Polymer 2015, 62, 28-38, doi:10.1016/j.polymer.2015.01.080.

6. Papadopoulos, L.; Klonos, P.A.; Tzetzis, D.; Papageorgiou, G.Z.; Kyritsis, A.; Bikiaris, D.N. Effects of graphene nanoplatelets on crystallization, mechanical performance and molecular dynamics of the renewable poly(propylene furanoate). Polymer 2020, 189, 122172, doi:10.1016/j.polymer.2020.122172.

7. Klonos, P.A.; Papadopoulos, L.; Terzopoulou, Z.; Papageorgiou, G.Z.; Kyritsis, A.; Bikiaris, D.N. Molecular dynamics in nanocomposites based on renewable poly(butylenes 2,5-furan-dicarboxylate) in situ 
reinforced by montmorillonite nanoclays: Effects of clay modification, crystallization, and hydration. J. Phys. Chem. B 2020, 124, 7306-7317, doi:10.1021/acs.jpcb.0c04306.

8. Sanusi, O.M.; Papadopoulos, L.; Klonos, P.A.; Terzopoulou, Z.; Aït Hocine, N.; Benelfellah, A.; Papageorgiou, G.Z.; Kyritsis, A.; Bikiaris, D.N. Calorimetric and dielectric study of renewable poly(hexylene 2,5-furan-dicarboxylate)-based nanocomposites in situ filled with small amounts of graphene platelets and silica nanoparticles. Polymers 2020, 12, 1239, doi:10.3390/polym12061239.

9. Kremer, F.; Schönhals, A. (Eds.) Broadband Dielectric Spectroscopy; Springer: Berlin, Germany, 2003; doi:10.1007/978-3-642-56120-7.

10. Klonos, P.A. Crystallization, glass transition, and molecular dynamics in PDMS of low molecular weights: A calorimetric and dielectric study. Polymer 2018, 159, 169-180, doi:10.1016/j.polymer.2018.11.028.

11. Klonos, P.; Terzopoulou, Z.; Koutsoumpis, S.; Zidropoulos, S.; Kripotou, S.; Papageorgiou, G.Z.; Bikiaris, D.; Kyritsis, A.; Pissis, P. Rigid amorphous fraction and segmental dynamics in nanocomposites based on poly(L-lactic acid) and nano-inclusions of 1-3D geometry studied by thermal and dielectric techniques. Eur. Polym. J. 2016, 82, 16-34, doi:10.1016/j.eurpolymj.2016.07.002.

12. Klonos, P.A.; Papadopoulos, L.; Papageorgiou, G.Z.; Kyritsis, A.; Pissis, P.; Bikiaris, D.N. Interfacial interactions, crystallization and molecular dynamics of renewable poly(propylene furanoate) in situ filled with initial and surface functionalized carbon nanotubes and graphene oxide. J. Phys. Chem. C 2020, 124, 10220-10234, doi:10.1021/acs.jpcc.0c01313.

13. Füllbrandt, M.; Purohit, P.J.; Schönhals, A. Combined FTIR and dielectric investigation of poly(vinyl acetate) adsorbed on silica particles. Macromolecules 2013, 46, 4626-4632, doi:10.1021/ma400461p.

14. Klonos, P.A.; Papadopoulos, L.; Tzetzis, D.; Kyritsis, A.; Papageorgiou, G.Z.; Bikiaris, D.N. Thermal, nanoindentation and dielectric study of nanocomposites based on poly(propylene furanoate) and various inclusions. Mater. Today Commun. 2019, 20, 100585, doi:10.1016/j.mtcomm.2019.100585.

15. Klonos, P.A.; Tegopoulos, S.N.; Koutsiara, C.S.; Kontou, E.; Pissis, P.; Kyritsis, A. Effects of CNTs on thermal transitions, thermal diffusivity and electrical conductivity in nanocomposites: Comparison between an amorphous and a semicrystalline polymer matrix. Soft Matter 2019, 15, 1813-1824, doi:10.1039/c8sm02478b.

16. Klonos, P.A.; Peoglos, V.; Bikiaris, D.N.; Kyritsis, A. Rigid amorphous fraction and thermal diffusivity in nanocomposites based on poly(L-lactic acid) filled with carbon nanotubes and graphene oxide. J. Phys. Chem. C 2020, 124, 5469-5479, doi:10.1021/acs.jpcc.9b11843.

17. Genovese, L.; Soccio, M.; Lotti, N.; Munari, A.; Szymczyk, A.; Paszkiewicz, S.; Linares, A.; Nogales, A.; Ezquerra, T.A. Effect of chemical structure on the subglass relaxation dynamics of biobased polyesters as revealed by dielectric spectroscopy: 2,5-furandicarboxylic acid vs. trans-1,4-cyclohexanedicarboxylic acid. Phys. Chem. Chem. Phys. 2018, 20, 15696-15706, doi:10.1039/C8CP01810C.

Publisher's Note: MDPI stays neutral with regard to jurisdictional claims in published maps and institutional affiliations.

(C) 2020 by the authors; licensee MDPI, Basel, Switzerland. This article is an open access article distributed under the terms and conditions of the Creative Commons by Attribution (CC-BY) license (http://creativecommons.org/licenses/by/4.0/). 